J. Indones. Math. Soc.

Vol. 19, No. 1 (2013), pp. 49-59.

\title{
ON SEMIPRIME SUBSEMIMODULES AND RELATED RESULTS
}

\author{
Hamid Agha Tavallaee ${ }^{1}$ and Masoud Zolfaghari ${ }^{2}$ \\ ${ }^{1}$ Department of Mathematics, Tehran-North Branch, \\ Islamic Azad University, Tehran, Iran \\ tavallaee@iust.ac.ir \\ ${ }^{1}$ Department of Mathematics, Semnan University, Semnan, Iran \\ m_zolfaghari@iust.ac.ir
}

\begin{abstract}
In this paper we introduce the notions of semiprime subsemimodules and semiprime $k$-subsemimodules and present some characterizations about them. Special attention has been paid, when semimodules are multiplication, to find extra properties of these semimodules. Moreover we prove a result for semiprime subsemimodules of quotient semimodules.
\end{abstract}

Key words: Semiprime subsemimodule, Strong semiprime $k$-subsemimodule, Ssemiring, S-semimodule. 